\title{
Prostate-specific Membrane Antigen-Based Nanomedicine Applications in the Diagnosis and Treatment of Prostate Cancer
}

\author{
(1) Deniz Bolat MD ${ }^{1,3}$, () Ayfer Haydaroğlu MD2,3 \\ 1 University of Health Sciences, Izmir Bozyaka Training and Research Hospital, Clinic of Urology, Izmir, Turkey \\ 2 Ege University Faculty of Medicine, Department of Radiation Oncology, Izmir, Turkey \\ ${ }^{3}$ Ege University Faculty of Medicine, Institute of Health Sciences, Department of Basic Oncology, Izmir, Turkey
}

\begin{abstract}
Nanomedicine is a branch of nanotechnology that includes the development of nanostructures and nanoanalytical systems for various medical applications. The rapid development of nanomedicine offers new possibilities in cancer diagnosis and treatment. New therapeutic strategies in cancer research using nanoparticles are being developed in order to improve the specificity and efficacy of drug delivery, thus reaching maximal effectiveness with minimal side effects. Due to its selective overexpression in prostate cancer (PCa), prostate-specific membrane antigen (PSMA) has been recognized as a highly promising target for diagnostic and therapeutic applications. This review provides an update on the PSMA-based nanomedicine applications in PCa.
\end{abstract}

Keywords: Prostate cancer, PSMA, nanomedicine, nanotechnology, theranostic concepts

\section{Introduction}

\section{Theranostic Concept}

Targeted cancer therapy can improve progression-free and overall survival. However, the greatest barrier to targeted therapy is identifying the patients who will benefit from it. Therefore, predictive markers are urgently needed. Radiolabeled probes can be used as predictive markers. For instance, target expression can be confirmed by positron-emitting tomography (PET) using F-18- or Ga-68-labeled ligands. The same ligands can be labeled with therapeutic radionuclides (Lu-177/Y-90) for radioligand therapy (RLT). This combination is called a theranostic pair.

Nuclear medicine specialists used this method with I-131 to treat metastatic thyroid adenocarcinoma in 1946 (1). By using I-131 in diagnostic screening, they were able to identify patients with differentiated thyroid carcinoma who would benefit from treatment with a higher dose of I-131 (2). In the 1990s, the theranostic approach progressed toward neuroendocrine tumors (NET). Somatostatin analogs DOTATOC, DOTANOC, and DOTATE were developed to selectively bind to NETs overexpressing somatostatin receptors (SSTR) $(3,4,5)$. Labeling these SSTR agonists with different radionuclides enabled the combination of diagnostic imaging (Ga-68) with radioligand treatment (Lu-177/Y-90) $(3,4,5)$. NETTER-1 was the first randomized prospective study comparing Lu-177labeled DOTATE with octreotide long-acting repeatable (LAR) in metastatic NET. Lu-DOTATE RLT significantly prolonged progression-free survival (PFS) compared to octreotide LAR (6). Progression was 4.8-fold more frequent among patients who received high-dose octreotide than in those given Lu-177DOTATE (HR: $0.21,95 \% \mathrm{Cl}: 0.13-0.34)(6)$. This treatment is currently known as peptide receptor radionuclide therapy (PRRT) and is widely accepted and used in patients with NET (5).

\section{Prostate-specific Membrane Antigen as a Target in Prostate Cancer}

Prostate-specific membrane antigen (PSMA), also known as glutamate carboxypeptidase II, is a promising theranostic target (7-9). PSMA is a type II transmembrane protein comprised of a small intracellular segment, a transmembrane domain, and an extracellular domain containing the catalytic site $(8,9)$. PSMA is expressed at low levels in various tissues such as prostate, brain, small intestine, and kidney $(8,9)$. While PSMA has different enzymatic functions in brain and small intestine, its enzymatic function in the prostate is not yet clear (9). Most importantly, PSMA is overexpressed in prostate cancer cells and its expression 
level is correlated with pathological grade $(10,11,12,13,14,15)$. Moreover, after ligand binding, PSMA is internalized via clathrincoated pits and endocytosis $(11,16)$. These characteristics have led to the development of therapeutic PSMA ligands labeled with different radionuclides.

\section{PSMA-targeted Agents}

One of the first probes that targeted PSMA was Indium-111 capromab pendetide (ProstaScint ${ }^{\circledR}$ ). Capromab is a monoclonal antibody that binds to the cytoplasmic domain of PSMA (16). Probes that target the extracellular domain of PSMA, such as antibody 1591, have been developed to improve tumor uptake. However, antibody-based approaches have limited diagnostic potential (8). In recent years, nanoparticle PSMA ligands have been developed. One of these is Ga-68-PSMA-11, which is the most commonly used PET probe in PSMA-based imaging (17). Ga-68-PSMA-11 shows excellent biodistribution and high tumor uptake (18). However, PSMA-11 cannot be labeled with Lu-177 or Y-90 for RLT (19). Therefore, nanoparticle ligands with different chelators were developed. Weineisen et al. $(19,20)$ synthesized DOTAGA-FFK (Sub-KuE), which can be labeled with Ga-68 or Lu-177/Y-90, and its optimized version, PSMA I\&T. PSMA I\&T has good dosimetry and similar biodistribution to Ga-68-PSMA-11 (20). A research group in Heidelberg also developed PSMA-617, which can be labeled with Ga-68 or Lu-177/Y-90. This probe showed high affinity to PSMA and high tumor base uptake $(21,22)$. Based on their similar biodistribution, PSMA-11 and PSMA-617 are frequently used in combination as diagnostic and therapeutic agents (22). Moreover, commercial ligands labeled with Tc-99m or I-131 have been developed as theranostic pairs $(23,24)$. Mease et al. (25) and Cho et al. (26) synthesized 18-FDCFBC, which is an F-18-labeled small-molecule PSMA inhibitor. This agent reliably detects prostate cancer. The second-generation probe 18-FDCFPyL has shown higher affinity for PSMA and tumor uptake than 18-FDCFBC (27). This probe has good dosimetry and biodistribution, but cannot be labeled with Lu-177 or Y-90 for RLT (28).

Here we present an overview of PSMA-targeted diagnosis and RLT.

\section{PSMA Imaging for Primary Diagnosis}

Imaging serves two purposes in the primary diagnosis of prostate cancer. The first is to detect disease progression in patients who have biopsy-proven disease or high metastasis risk, and the second is to determine primary tumor location in patients with high suspicion but negative biopsy (29). Magnetic resonance imaging (MRI) is currently the preferred modality for T staging (29). T2-weighted, dynamic contrast-enhanced, and diffusionweighted sequences are used to identify tumor involvement, extracapsular extension, seminal vesicle invasion, and/or other organ involvement. Moreover, combining these protocols with multiparametric MRI (MP-MRI) enables the differentiation of benign and malignant prostate tissue (30). MRI is superior to C-11-choline PET/CT (31,32,33), FDG-18 PET/CT (31), and ultrasound-guided biopsy (34) for primary diagnosis.

\section{PSMA PET Imaging for T Staging}

Rowe et al. (35) reported that MRI had higher sensitivity in the detection of primary lesions compared to F-18-DCFBC PET/CT. The importance of the second-generation radionuclide tracer F-18-DCFPyL in the detection of primary prostate lesions has not yet been evaluated. Ga-68-PSMA-11 PET is superior to MP-MRI, with a sensitivity of $49-76 \%$ in different populations $(36,37)$. Ga-68-PSMA-11 uptake was found to be significantly higher in histopathology-positive areas than negative areas (37). The accuracy of GA-68-PSMA-11 PET/CT in the detection of seminal vesicle invasion and extracapsular tumor spread was $86 \%$ and $71 \%$, respectively (37). Based on these findings, PSMA imaging has the potential to replace MP-MRI for determining tumor location.

Clinically, treatment options for localized prostate cancer may vary from active surveillance to radiotherapy and radical prostatectomy. $T$ staging is important for determining the best approach. European Association of Urology guidelines recommend MP-MRI for T staging (29), and although PSMA is superior in the detection of primary prostatic lesions, increased diagnostic accuracy has not been shown to have a significant effect on patient management. Of 15 patients who underwent MRI and were diagnosed with prostate cancer, planned radiotherapy was changed in $26.4 \%$ after additional PSMA imaging (additional dose, wide area) (38). However, due to inadequate long-term follow up and lack of a control group, the effects of these findings on patient outcomes are not known. Therefore, further studies are required to determine whether PSMA PET influences clinical management at initial diagnosis of patients with prostate cancer.

\section{PSMA Imaging for $\mathbf{N}$ Staging}

Many studies have demonstrated high reliability of Ga-68PSMA-11 PET/CT in N-staging at primary diagnosis. Budäus et al. (39) retrospectively compared lymph node findings in preoperative Ga-68-PSMA-11 PET/CT with histopathology in 12 patients and reported a low detection rate of $33.3 \%$ and mean sizes of detectable and undetectable lymph nodes of $13.6 \mathrm{~mm}$ and $4.3 \mathrm{~mm}$, respectively. Later studies showed that Ga-68-PSMA-11 PET/CT or PET/MRI were superior to conventional imaging techniques in the detection of lymph nodes $(40,41,42)$. In one study, lymph node metastasis was detected using Ga-68-PSMA-11 PET/CT in 12 patients in whom conventional imaging modalities did not show lymph node involvement (40). Herleman et al. (41) demonstrated that the accuracy of Ga-68-PSMA-11 PET/CT (88\%) was superior to that of CT $(77 \%)$. More importantly, $40 \%$ of the lymph nodes detected via Ga-68-PSMA-11 PET/CT were reported to have short axis lengths of $<5 \mathrm{~mm}(41)$. In a prospective study including 30 moderate/high-risk patients, the mean diameters of lymph nodes that were actually detected and those that could not be detected using Ga-68-PSMA-11 PET/CT were $4.7 \mathrm{~mm}$ and $2.7 \mathrm{~mm}$, respectively (42). None of the currently available imaging modalities are able to accurately detect lymph nodes because of their size. However, PSMA PET is superior to conventional imaging methods in the detection of lymph nodes. With the exception of the study by Maurer et al. (40), its 
specificity and sensitivity were higher than CT and MRI. PSMA PET imaging has the potential to determine $\mathrm{N}$ stage and thus to change initial prostate cancer stage.

\section{PSMA Imaging for M Staging}

In all previous studies, Ga-68-PSMA-11 PET/CT was used for whole-body screening. PSMA imaging detects bone and visceral metastases more accurately than conventional imaging modalities (CT and bone scintigraphy). In a retrospective study including 126 patients, Ga-68-PSMA-11 PET/CT detected osseous metastases with $99 \%$ sensitivity and $88 \%$ specificity, whereas these rates were $87 \%$ and $61 \%$ in bone scintigraphy, respectively (43).

Although PSMA PET imaging is better in M staging, it is not yet clear whether this advantage makes a positive impact in patient management.

\section{Limitations of PSMA PET in Primary Staging}

Firstly, PSMA expression in primary lesions is variable and heterogeneous, and thus, sensitivity is limited (44). Moreover, benign diseases such as prostate hyperplasia are associated with high PSMA expression and have the potential to reduce specificity $(45,46)$. Although PSMA PET is more reliable in TNM staging compared to conventional methods, studies focusing on the clinical effect of Ga-68-PSMA-11 PET/CT at primary diagnosis are needed.

\section{PSMA Imaging for Biochemical Recurrence}

The risk of biochemical recurrence of prostate cancer is $15-20 \%$ within 5 years of first treatment and $25-30 \%$ within 10 years $(47,48)$. Correct diagnosis and tumor location are essential because clinical management varies from active surveillance to local/systemic treatment (29). Despite important advances in imaging methods, determining the location of recurrences remains a major challenge. For patients with elevated PSA or clinical symptoms, current guidelines recommend either radionuclide bone scintigraphy, abdominopelvic CT, MP-MRI, or choline/acetate PET/CT (29). However, the recommended imaging modalities have limited detection rates $(29,24)$. In patients with serum PSA level $<7 \mathrm{ng} / \mathrm{mL}$, the probability of having a positive bone scintigraphy is $<5 \%(29,30,31,32,33,3$ $4,35,36,37,38,39,40,41,42,43,44,45,46,47,48,49,50,51)$. CT is positive in only $11-14 \%$ of patients with biochemical recurrence (51). Moreover, in a study of 132 patients, it was reported that in order for CT to be positive, the mean PSA value must be $27.4 \mathrm{ng} / \mathrm{mL}$ (51). In patients with high-risk prostate cancer, MRI and choline PET/CT were reported to have comparable sensitivity in the detection of bone metastasis (52). However, the rate of lymph node detection is very low (53). Depending on serum PSA levels, choline or acetate PET are reported to have a detection rate between $11-75 \%$, and the detection range is $5-44 \%$ at PSA $<1 \mathrm{ng} / \mathrm{mL}(54,55,56,57,58,59,60,61)$. Its main limitation is low sensitivity for micrometastatic disease. Therefore, choline PET/CT is now recommended for patients with biochemical recurrence and PSA $>1 \mathrm{ng} / \mathrm{mL}$ (29). Ga-68PSMA-11 PET/CT is promising for determining tumor location in patients with biochemical recurrence. There are studies reporting a high overall accuracy rate of around $54-100 \%$ for median PSA levels of $0.2-4.6 \mathrm{ng} / \mathrm{mL}$. Moreover, the detection rate in patients with PSA level $<1 \mathrm{ng} / \mathrm{mL}$ is between $44-73 \%$. These results are also confirmed by a prospective study including 31 patients. Location of recurrence was accurately determined in 22/31 patients (71\%) using Ga-68-PSMA-11 PET/CT (62). In this patient group, median PSA was reported as $2.0 \mathrm{ng} / \mathrm{mL}(0.1$ $130 \mathrm{ng} / \mathrm{mL}$ ). The detection rate was found to be $47.6 \%$ in those with PSA $<0.83 \mathrm{ng} / \mathrm{mL}$ (62). The rate of detection by Ga-68PSMA-11 PET/CT increased with elevated PSA levels (63-65). However, Gleason score and neoadjuvant or adjuvant androgen therapy did not affect the detection rate (63).

\section{Impact on Patient Management}

Ga-68-PSMA-11 PET/CT has resulted in management changes in patients with early biochemical recurrence $(65,66)$. In two different studies, management of treatment was changed in $60 \%$ and $29 \%$ of patients scheduled for radiotherapy based on the results of Ga-68-PSMA-11 PET/CT $(65,66)$. In one of those studies, Sterzing et al. (66) reported a switch from radiotherapy to systemic treatment in only 4 of 42 patients $(10 \%)$ and changes in radiation dosage and location only in the other 21 of 42 patients (50\%). In the other, Van Leeuwen et al. (65) reported significant management changes in $11 / 20$ patients (55\%) who were scheduled for salvage radiotherapy. Of these patients, therapy was changed from radiotherapy to surgery in $1 / 20(5 \%)$, androgen suppression treatment was added for $6 / 20(30 \%)$, salvage radiotherapy was changed to extrapelvic stereotactic radiotherapy in $3 / 20(15 \%)$, and stereotactic radiotherapy was added for an extrapelvic lesion in 1/20 (5\%) (65). Overall, evidence to date shows that PSMA imaging has overcome the limitations of choline PET/CT and conventional imaging modalities in patients with biochemical recurrence and low PSA levels. In addition, PSMA imaging influences treatment approach in some patients with biochemical recurrence. Therefore, PSMA imaging has the potential to become routine for patients with biochemical recurrence due to its superiority over conventional imaging modalities and its role in guiding therapeutic management (66).

\section{PSMA Therapy}

Current approaches in metastatic castration-resistant prostate cancer include chemotherapy, hormone therapy, and abiraterone or enzalutamide. In addition, Radium-223 was approved for the treatment of symptomatic bone metastases. The first RLT in prostate cancer used Lu-177-J591, a monoclonal antibody with affinity for the extracellular domain of PSMA (67). Although this treatment showed promising outcomes, it was limited due to myelosuppression (67). With the development of PSMA ligands with nanoparticles, Lu-177-based radionuclide therapies are being reinvestigated in patients with metastatic prostate cancer. Some studies have yielded promising results using Lu-177labeled PSMA ligands. The mean tumor dose is 6-12-fold higher than in the critical organs, kidneys, and salivary glands (68). Moreover, the tumor/organ ratio is higher than Lu-177DOTATE, which is the standard RLT for NET patients $(68,69)$. Reduction in PSA was observed in $59-89 \%$ of patients after a single dose of Lu-177-PSMA RLT using PSMA-617 or PSMA I\&T. In addition, the reduction in PSA was greater than 50\% 
in $26.3-58.9 \%$ of the patients. Furthermore, Ga-68-PSMA PET/ CT was performed on patients at 6 months after the last cycle to determine disease progression. Based on different criteria, partial treatment response was seen in $56-91 \%$, stable disease in $0-64 \%$, and progression in $9.1-36 \%$. Finally, overall survival was compared in patients under Lu-177-PSMA treatment among a cohort receiving best supportive therapy. It was reported that overall survival was 29.4 weeks with Lu-177-PSMA treatment versus 19.4 weeks with best supportive care (HR: 0.44, 95\% $\mathrm{Cl}$ 0.20-0.95, $\mathrm{P}=0.031$ ) (70). In conclusion, Lu-177-PSMA can potentially prolong life. A similar effect was shown in advanced prostate cancer using I-131-labeled MIP-1095 compound, but the data are limited (24).

\section{Toxicity}

Patients receiving Lu-177-labeled PSMA RLT experienced severe side effects. Mild and reversible side effects noted in retrospective studies included dry mouth, nausea, and fatigue $(70,71,72,73,74,75,76)$. Heck et al. (74) reported grade 1-2 toxicity such as anemia (32\%) and thrombocytopenia (25\%). In another study, grade 3 anemia occurred in 2/24 patients (8.3\%) (72). No marked nephrotoxicity (grade 3,4) was observed $(71,72,73,74,75,76)$. Most patients tolerated therapy, and no acute side effects after Lu-177-PSMA injection have been reported $(72,73,74,75,76)$. Ahmadzadehfar et al. (72) retrospectively studied adverse events in 10 patients and reported grade 3/4 hematological toxicity in 1 patient 7 weeks after RLT administration. Most patients $(n=6)$ showed no hematological toxicity during the 8 weeks after injection (72). When compared with current chemotherapies, Lu-177based therapies have milder side effects. In the GETUG-AFU 15 study, $38 \%$ of patients receiving chemotherapy together with androgen suppression treatment experienced severe side effects, primarily neutropenia (77).

\section{Conclusion}

Lu-177-PSMA therapy has shown promising results. It is efficient and well-tolerated, and can prolong overall survival. However, most studies have been retrospective in design. Further randomized, controlled studies are needed to demonstrate the clinical value of PSMA-targeted RLT.

\section{Ethics}

Peer-review: Externally peer-reviewed.

\section{Authorship Contributions}

Consept: A.H., Design: D.B., A.H., Data Collection or Processing: D.B., Analysis or Interpretation: A.H., Literature Search: D.B., A.H., Writing: D.B.

Conflict of Interest: No conflict of interest was declared by the authors.

Financial Disclosure: The authors declared that this study received no financial support.

\section{References}

1. Seidlin SM, Marinelli LD, Oshry E. Radioactive iodine therapy; effect on functioning metastases of adenocarcinoma of the thyroid. J Am Med Assoc 1946;132:838-847.
2. Robbins RJ, Schlumberger MJ. The evolving role of (131)I for the treatmentof differentiated thyroid carcinoma. I Nucl Med 2005;46(Suppl 1):28-37.

3. Baum RP, Kulkarni HR. THERANOSTICS: From Molecular Imaging Using Ga-68 Labeled Tracers and PET/CT to Personalized Radionuclide Therapy - The Bad Berka Experience. Theranostics 2012;2:437-447.

4. Kulkarni H, Baum RP. Molecular Imaging Using PET/CT Applying 68Ga-Labeled Tracers and Targeted Radionuclide Therapy: Theranostics on the Way to Personalized Medicine. J Postgrad Med Edu Res 2013;47:47-53.

5. Kwekkeboom DJ, Kam BL, van Essen M, et al. Somatostatin Receptor-based Imaging and Therapy of Gastroenteropancreatic Neuroendocrine Tumors. Endocr Relat Cancer 2010;17:53-73.

6. Strosberg J, Wolin E, Chasen B, et al. 6LBA 177-Lu-Dotatate Significantly Improves Progression-free Survival in Patients with Midgut Neuroendocrine Tumours: Results of the Phase III NETTER-1 Trial. Eur J Cancer 2015;51(Suppl 3):710.

7. Lütje S, Heskamp S, Cornelissen AS, et al. PSMA Ligands for Radionuclide Imaging and Therapy of Prostate Cancer: Clinical Status. Theranostics 2015;5:1388-1401.

8. Denmeade S. Prostate-Specific Membrane Antigen. In: Schwab M, ed. Encyclopedia of Cancer. Springer, Berlin, Heidelberg, 2011.p. 2452-2455.

9. Rahbar K, Afshar-Oromieh A, Jadvar H, Ahmadzadehfar H. PSMA Theranostics: Current Status and Future Directions. Mol Imaging 2018;17:1536012118776068.

10. Liu T, Wu LY, Kazak M, Berkman CE. Cell-Surface Labeling and Internalization by a Fluorescent Inhibitor of Prostate-specific Membrane Antigen. Prostate 2008;68:955-964.

11. Perner $S$, Hofer MD, Kim R, et al. Prostate-specific membrane antigen expression as a predictor of prostate cancer progression. Hum Pathol 2007;38:696-701.

12. Mannweiler S, Amersdorfer P, Trajanoski S, et al. Heterogeneity of prostate-specific membrane antigen [PSMA] expression in prostate carcinoma with distant metastasis. Pathol Oncol Res 2009;15:167-172.

13. Bostwick DG, Pacelli A, Blute $M$, et al. Prostate specific membrane antigen expression inprostatic intraepithelial neoplasia and adenocarcinoma: a study of 184 cases. Cancer 1998;82:2256-2261.

14. Ross JS, Sheehan CE, Fisher HA, et al. Correlation of primary tumor prostate-specific membrane antigen expression with disease recurrence in prostate cancer. Clin Cancer Res 2003;9:6357-6362.

15. Rajasekaran SA, Anilkumar G, Oshima E, et al. A novel cytoplasmic tail MXXXL motif mediates the internalization of prostate-specific membrane antigen. Mol Biol Cell 2003;14:4835-4845.

16. Holmes EH. PSMA Specific Antibodies and Their Diagnostic and Therapeutic Use. Expert Opin Investig Drugs 2001;10:511-519.

17. Eder M, Schäfer M, Bauder-Wüst U, et al. $68 \mathrm{Ga}$-Complex Lipophilicity and the Targeting Property of a Urea-Based PSMA Inhibitor for PET Imaging. Bioconjug Chem 2012;23:688-697.

18. Afshar-Oromieh $A$, Malcher $A$, Eder $M$, et al. PET imaging with a [68Ga]gallium-labelled PSMA ligand for the diagnosis of prostate cancer: biodistribution in humans and first evaluation of tumour lesions. Eur J Nucl Med Mol Imaging 2013;40:486-495.

19. Weineisen M, Simecek J, Schottelius $M$,et al. Synthesis and PreclinicalEvaluation of DOTAGA-conjugated PSMA Ligands for Functional Imaging and Endoradiotherapy of Prostate Cancer. EJNMMI Res 2014;4:63.

20. Weineisen M, Schottelius M, Simecek J, et al. 68Ga- and 177LuLabeled PSMA I\&T: Optimization of a PSMA-Targeted Theranostic Concept and First Proof-of-Concept Human Studies. J Nucl Med 2015;56:1169-1176. 
21. Benešová $M$, Schäfer $M$, Bauder-Wüst $U$, et al. Preclinical Evaluation of a Tailor-Made DOTA-Conjugated PSMA Inhibitor with Optimized Linker Moiety for Imaging and Endoradiotherapy of Prostate Cancer. J Nucl Med 2015;56:914-920.

22. Afshar-Oromieh A, Hetzheim $\mathrm{H}$, Kratochwil $\mathrm{C}$, et al. The Theranostic PSMA Ligand PSMA-617 in the Diagnosis of Prostate Cancer by PET/CT: Biodistribution in Humans, RadiationDosimetry, and First Evaluation of Tumor Lesions. J Nucl Med 2015;56:1697-1705.

23. Vallabhajosula S, Nikolopoulou A, Babich JW, et al. $99 \mathrm{mTC}-$ Labeled Small-Molecule Inhibitors of Prostate-Specific Membrane Antigen: Pharmacokinetics and Biodistribution Studies in Healthy Subjects and Patients with metastatic prostate cancer. J Nucl Med 2014;55:1791-1798.

24. Zechmann CM, Afshar-Oromieh A, Armor $T$, et al. Radiation Dosimetry and First Therapy Results with a (124)I/ (131)I-labeled Small Molecule (MIP-1095) Targeting PSMA for Prostate Cancer Therapy. Eur J Nucl Med Mol Imaging 2014;41:1280-1292.

25. Mease RC, Dusich CL, Foss CA, et al. N-[N-[[S]-1,3-Dicarboxypropyl] carbamoyl]-4-[18F]fluorobenzyl-L-cysteine, [18F]DCFBC: a new imaging probe for prostate cancer. Clin Cancer Res 2008;14:3036-3043.

26. Cho SY, Gage KL, Mease RC, et al. Biodistribution, tumor detection, and radiation dosimetry of $18 \mathrm{~F}-\mathrm{DCFBC}$, a low-molecular weight inhibitor of prostate-specific membrane antigen, in patients with metastatic prostate cancer. J Nucl Med 2012;53:1883-1891.

27. Chen Y, Pullambhatla M, Foss CA, et al. 2-[3-\{1-Carboxy-5-[[6-[18F] fluoro-pyridine-3-carbonyl]-amino]-pentyl\}-urei do]-pentanedioic acid, [18F]DCFPyL, a PSMA-based PET imaging agent for prostate cancer. Clin Cancer Res 2011;17:7645-7653.

28. Szabo Z, Mena E, Rowe SP,et al. Initial Evaluation of [18F]DCFPyL for Prostate-Specific Membrane Antigen [PSMA]-Targeted PET Imaging of Prostate Cancer. Mol Imaging Biol 2015;17:565-574.

29. Mottet N, Bellmunt J, Bolla M, et al. EAU-ESTRO-SIOG Guidelines on Prostate Cancer. Part 1: Screening, Diagnosis, and Local Treatment with Curative Intent. Eur Urol 2017;71:618-629.

30. Bratan F, Niaf E, Melodelima C, et al. Influence of Imaging and Histological Factors on Prostate Cancer Detection and Localisation on Multiparametric MRI:A Prospective Study. Eur Radiol 2013;23:2019-2029.

31. Mapelli P, Picchio M. Initial Prostate Cancer Diagnosis and Disease Staging--the Role of Choline-PET-CT. Nat Rev Urol 2015;12:510-518.

32. Testa C, Schiavina R, Lodi R, et al. Prostate cancer: sextant localization with MR imaging, MR spectroscopy, and 11C-choline PET/CT. Radiology 2007;244:797-806.

33. Van den Bergh $L$, Koole $M$, Isebaert $S$, et al. Is there an additional value of 11C-choline PET/CTto T2-weighted MRI images in the localization of intraprostatic tumor nodules? Int J Radiat Oncol Biol Phys 2012;83:1486-1492.

34. Sonn GA, Chang E, Natarajan S, et al. Value of targeted prostate biopsy using magneticresonance-ultrasound fusion in men with prior negative biopsy and elevated prostatespecific antigen. Eur Urol 2014;65:809-815.

35. Rowe SP, Gage KL, Faraj SF, et al. 18F-DCFBC PET/CT for PSMA-Based Detection and Characterization of Primary Prostate Cancer. J Nucl Med 2015;56:1003-1010.

36. Rhee H, Thomas P, Shepherd B, et al. Prostate Specific Membrane Antigen Positron Emission Tomography May Improve the Diagnostic Accuracy of Multiparametric Magnetic Resonance Imaging in Localized Prostate Cancer. J Urol 2016;196:1261-1267.

37. Fendler WP, Schmidt DF, Wenter V, et al. 68Ga-PSMA-HBED-CC PET/ CT Detects Location and Extent of Primary Prostate Cancer. J Nucl Med 2016;57:1720-1725.

38. van Leeuwen PJ, Stricker P, Hruby G, et al. (68) Ga-PSMA has a high detection rate of prostatecancer recurrence outside the prostatic fossa in patients being considered for salvage radiation treatment. BJU Int 2016;117:732-739.
39. Budäus L, Leyh-Bannurah SR, Salomon G, et al. Initial Experience of (68)Ga-PSMA PET/CT Imaging in High-risk Prostate Cancer Patients Prior to Radical Prostatectomy. Eur Urol 2016;69:393-396.

40. Maurer T, Gschwend JE, Rauscher I, et al. Diagnostic Efficacy of (68)Gallium-PSMA Positron Emission Tomography Compared to Conventional Imaging for Lymph Node Staging of 130 Consecutive Patients with Intermediate to High Risk Prostate Cancer. J Urol 2016;195:1436-1443.

41. Herlemann A, Wenter V, Kretschmer A, et al. 68Ga-PSMA Positron Emission Tomography/Computed Tomography Provides Accurate Staging of Lymph Node Regions Prior to Lymph Node Dissection in Patients with Prostate Cancer. Eur Urol 2016;70:553-557.

42. van Leeuwen PJ, Emmett L, Ho B, et al. Prospective evaluation of 68Gallium-prostate-specific membrane antigen positron emission tomography/computed tomography for preoperative lymph node staging in prostate cancer. BJU Int 2017; 119:209-215.

43. Pyka T, Okamoto S, Dahlbender $M$, et al. Comparison of Bone Scintigraphy and 68Ga-PSMA PET for Skeletal Staging in Prostate Cancer. Eur J Nucl Med Mol Imaging 2016;43:2114-2121.

44. Mannweiler S, Amersdorfer P, Trajanoski S, et al. Heterogeneity of Prostate-Specific Membrane Antigen [PSMA] Expression in Prostate Carcinoma with Distant Metastasis. Pathol Oncol Res 2009;15:167-172.

45. Ben Jemaa A, Bouraoui Y, Sallami S, et al. Co-expression and Impact of Prostate Specific Membrane Antigen and Prostate Specific Antigen in Prostatic Pathologies. J Exp Clin Cancer Res 2010;29:171.

46. Beckett ML, Cazares LH, Vlahou A, et al. Prostate-specific membrane antigen levels in sera from healthy men and patients with benign prostate hyperplasia or prostate cancer. Clin Cancer Res 1999;5:4034-4040.

47. Han M, Partin AW, Zahurak M, et al. Biochemical (Prostate Specific Antigen) Recurrence Probability Following Radical Prostatectomy for Clinically Localized Prostate Cancer. J Urol 2003;169:517-523

48. Amling CL, Blute ML, Bergstralh EJ, et al. Long-Term Hazard of Progression After Radical Prostatectomy For Clinically Localized Prostate Cancer: Continued Risk of Biochemical Failure After 5 Years. J Urol 2000;164:101-105.

49. Beresford MJ, Gillatt D, Benson RJ, Ajithkumar T. A systematic review of the role of imaging before salvage radiotherapy for postprostatectomy biochemical recurrence. Clin Oncol (R Coll Radiol) 2010;22:46-55.

50. Gomez P, Manoharan M, Kim SS, Soloway MS. Radionuclide bone scintigraphy in patients with biochemical recurrence after radical prostatectomy: when is it indicated? BJU Int 2004;94:299-302.

51. Kane CJ, Amling CL, Johnstone PA, et al. Limited value of bone scintigraphy and computed tomography in assessing biochemical failure after radical prostatectomy. Urology 2003;61:607-611.

52. Luboldt W, Küfer R, Blumstein N, et al. Prostate carcinoma: diffusionweighted imaging as potential alternative to conventional MR and 11C-choline PET/CT for detection of bone metastases. Radiology 2008;249:1017-1025.

53. Budiharto $T$, Joniau $S$, Lerut $E$, et al. Prospective evaluation of 11C-choline positron emission tomography/computed tomography and diffusion-weighted magnetic resonance imaging for the nodal staging of prostate cancer with a high risk of lymph node metastases. Eur Urol 2011;60:125-130.

54. Giovacchini G, Picchio $M$, Briganti $A$, et al. [11C]choline positron emission tomography/computerized tomography to restage prostate cancer cases with biochemical failure after radical prostatectomy and no disease evidence on conventional imaging. J Urol 2010;184:938-943.

55. Giovacchini G, Picchio M, Coradeschi E, et al. Predictive factors of [[11]C]choline PET/CT in patients with biochemical failure after radical prostatectomy. Eur J Nucl Med Mol Imaging 2010;37:301-309. 
56. Fuccio C, Castellucci P, Schiavina R, et al. Role of 11C-choline PET/ $\mathrm{CT}$ in the re-staging of prostate cancer patients with biochemical relapse and negative results at bone scintigraphy. Eur J Radiol 2012;81:893-896.

57. Mitchell CR, Lowe VJ, Rangel LJ, et al. Operational characteristics of [11]c-choline positron emission tomography/computerized tomography for prostate cancer with biochemical recurrence after initial treatment. J Urol 2013;189:1308-1313.

58. Soyka JD, Muster MA, Schmid DT, et al. Clinical impact of 18 F-choline $\mathrm{PET} / \mathrm{CT}$ in patients with recurrent prostate cancer. Eur J Nucl Med Mol Imaging 2012;39:936-943.

59. Ceci F, Castellucci P, Mamede M, et al. [11]C-Choline PET/CT in patients with hormone resistant prostate cancer showing biochemical relapse after radical prostatectomy. Eur J Nucl Med Mol Imaging 2013;40:149-155.

60. Rybalov M, Breeuwsma AJ, Leliveld AM, et al. Impact of total PSA, PSA doubling time and PSA velocity on detection rates of 11C-Choline positron emission tomography in recurrent prostate cancer. World J Urol 2013;31:319-323.

61. Castellucci P, Fuccio C, Nanni C, et al. Influence of trigger PSA and PSA kinetics on 11C-Choline PET/CT detection rate in patients with biochemical relapse after radical prostatectomy. J Nucl Med 2009;50:1394-1400.

62. Ceci F, Uprimny C, Nilica B, et al. 68Ga-PSMA PET/CT for Restaging Recurrent Prostate Cancer: Which Factors Are Associated with PET/CT Detection Rate? Eur J Nucl Med Mol Imaging 2015;42:1284-1294.

63. Eiber M, Maurer T, Souvatzoglou M, et al. Evaluation of Hybrid 68GaPSMA Ligand PET/CTin 248 Patients with Biochemical Recurrence After Radical Prostatectomy. J Nucl Med 2015;56:668-674.

64. Sachpekidis C, Eder M, Kopka K, et al. 68Ga-PSMA-11 Dynamic PET/ CT Imaging inBiochemical Relapse of Prostate Cancer. Eur J Nucl Med Mol Imaging 2016;43:1288-1299.

65. van Leeuwen PJ, Stricker P, Hruby G, et al. 68Ga-PSMA Has High Detection Rate of Prostate Cancer Recurrence outside the Prostatic Fossa in Patients Being Considered forSalvage Radiation Treatment. BJU Int 2016;117:732-739.

66. Sterzing F, Kratochwil C, Fiedler $\mathrm{H}$, et al. 68Ga-PSMA-11 PET/CT: A New Technique with High Potential for the Radiotherapeutic Management of Prostate Cancer Patients. Eur J Nucl Med Mol Imaging 2016;43:34-41.
67. Lütje S, Slavik R, Fendler W, et al. PSMA ligands in prostate cancer - Probe optimization and theranostic applications. Methods 2017; 130:42-50.

68. Fendler WP, Reinhardt S, Ilhan $H$, et al. Preliminary experience withdosimetry, response and patient reported outcome after $177 \mathrm{Lu}$ PSMA-617 therapy formetastatic castration-resistant prostate cancer. Oncotarget 2017;8:3581-3590.

69. Gupta SK, Singla S, Thakral P, Bal CS. DosimetricAnalyses of Kidneys, Liver, Spleen, Pituitary Gland, and Neuroendocrine Tumors of Patients Treated With 177Lu-DOTATATE. Clin Nucl Med 2013;38:188-194.

70. Ahmadzadehfar H, Eppard E, Kürpig S, et al. Therapeutic Response and Side Effects of Repeated Radioligand Therapy with 177LuPSMA-DKFZ-617 of Castrate-resistant Metastatic Prostate Cancer. Oncotarget 2016;7:12477-12488.

71. Rahbar K, Bode A, Weckesser M, et al. Radioligand Therapy With177Lu-PSMA-617 as A Novel Therapeutic Option in Patients With Metastatic Castration Resistant Prostate Cancer. Clin Nucl Med 2016;41:522-528.

72. Ahmadzadehfar $H$, Rahbar K, Kürpig $S$, et al. Early side effects and first results of radioligand therapy with (177)Lu-DKFZ-617 PSMA of castrate-resistant metastatic prostate cancer: a two-centre study. EJNMMI Res 2015;5:114.

73. Baum RP, Kulkarni HR, Schuchardt C, et al. Lutetium-177 PSMA Radioligand Therapy of Metastatic Castration-Resistant Prostate Cancer: Safety and Efficacy. J Nucl Med 2016;57:1006-1013.

74. Heck MM, Retz M, D'Alessandria C, et al. Systemic Radioligand Therapy with 177Lu Labeled Prostate Specific Membrane Antigen Ligand forlmaging and Therapy in Patients with Metastatic Castration Resistant Prostate Cancer. J Urol 2016;196:382-391.

75. KratochwilC, Giesel FL, Stefanova M, etal.PSMA-targeted Radionuclide Therapy of Metastatic Castration-resistant Prostate Cancer with Lu177 Labeled PSMA-617. J Nucl Med 2016;57:1170-1176.

76. Rahbar K, Schmidt M, Heinzel A, et al. Response and Tolerability of a Single Dose of 177Lu-PSMA-617 in Patients with Metastatic Castration-resistant Prostate Cancer: A Multicenter Retrospective Analysis. J Nucl Med 2016;57:1334-1338.

77. Gravis G, Fizazi K, Joly F, et al. Androgen-deprivation therapy alone or with docetaxel in non-castrate metastatic prostate cancer (GETUGAFU 15): a randomised, open-label, phase 3 trial. Lancet Oncol 2013;14:149-158. 